de um programa de Gestão do conhecimento em uma instituição de pesquisa e inovação. Para tanto, serão utilizadas duas ferramentas para a elaboração do diagnóstico: a sociometria e a Organizational Learning Survey.

A primeira ferramenta foi selecionada pelo seu poder de explicitar os meios informais de comunicação e transmissão de informações e conhecimentos, por meio da identificação da atração/rejeição dos indivíduos, bem como, a ordenação de grupos de trabalho para melhor gerir o conhecimento dentro deles, Santos (2004).

Já a segunda ferramenta é a Organizational Learning Survey, e foi selecionada por permitir uma análise quantitativa que considera todo o conjunto de perguntas, bem como, sua estrutura de correlação, dessa forma fornecendo o cálculo do potencial de aprendizagem organizacional (PAPO) da instituição.

O Laboratório Nacional de Astrofisica - LNA, é o objeto de estudo selecionado por ser uma instituição orientada à inovação e à geração de novos conhecimentos, e possui posição de liderança nacional em ciência e tecnologia, o que lhe permite a introdução de trabalhos voltados à GC.

Este artigo está organizado em oito tópicos: no primeiro se encontra uma breve introdução quanto aos objetivos da pesquisa; o segundo e terceiro tópicos tratam de uma revisão da literatura; o quarto, refere-se aos instrumentos de pesquisa; o quinto e sexto tópicos relatam a estrutura da pesquisa e seus resultados; e finalmente o sétimo e oitavo tópicos relatam as considerações finais do artigo e a bibliografia utilizada.

\section{A gestão do conhecimento}

Segundo Drucker (1993), o recurso básico para a economia será o conhecimento, assim, é de suma importância gerir este conhecimento em benefício das organizações. Nonaka e Takeuchi (1997) afirmam que a geração do conhecimento dentro de uma organização ocorre em duas dimensões, o Conhecimento Tácito e o Conhecimento Explícito. O Quadro 1 ilustra a distinção dos conceitos.

Dessa forma, o processo de conversão do conhecimento ocorre:

- De Tácito em Tácito, denominada Socialização: É o processo de compartilhamento do conhecimento e experiências, tais como, modelos mentais, habilidades e técnicas.

- De Tácito em Explícito, denominado Externalização: Criado à medida que o conhecimento tácito se torna explícito tais como analogias, conceitos, hipóteses ou modelos.

- De Explícito em Explícito, Combinação: a conversão do conhecimento requer a combinação de diferentes conjuntos de conhecimentos sistêmicos.

- De Explícito em Tácito, denominada Internalização: É a incorporação do conhecimento explícito ao conhecimento tácito por meio prático operacional.

Em decorrência de tais interações, a transferência do conhecimento surge em forma de espiral, contínua e crescente, o que permite a transferência, criação e inovação do conhecimento, gerando vantagens competitivas (Figura 1).

Porém, Li e Gao (2003) afirmam que tal teoria não considera o real nível do conhecimento tácito presente nas empresas e que a criação do conhecimento deve, primeiro, ocorrer com a transferência de conhecimentos tácitos, de forma que novos conceitos sejam criados, gerando inovação, apoiada pela aprendizagem contínua e culminando em vantagens competitivas.

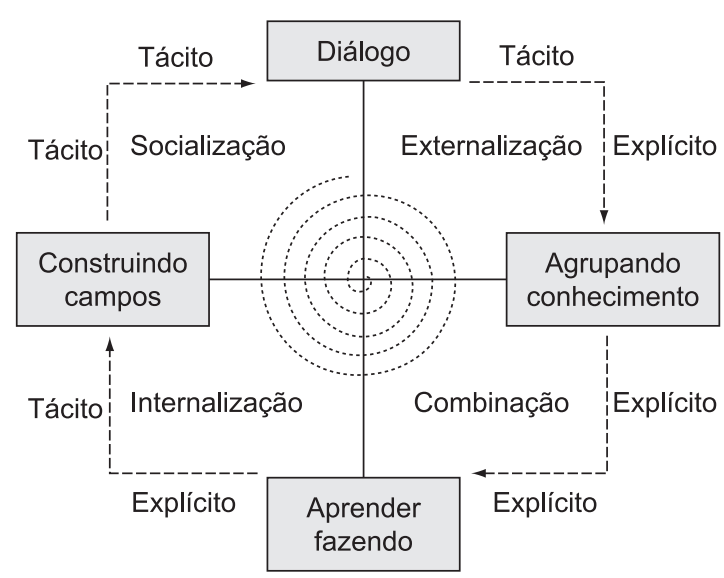

Figura 1. Transformação do conhecimento. Fonte: Nonaka e Takeuchi (1997).

Quadro 1. Duas dimensões do conhecimento.

\begin{tabular}{|cc|}
\hline Conhecimento tácito (subjetivo) & Conhecimento explícito (objetivo) \\
\hline Conhecimento da experiência (corpo) & Conhecimento da racionalidade (mente) \\
Conhecimento simultâneo (aqui e agora) & Conhecimento seqüencial (lá e então) \\
Conhecimento análogo (prática) & Conhecimento digital (teoria) \\
\hline
\end{tabular}

Fonte: Nonaka e Takeuchi (1997). 
Leonard e Barton (1992) afirmam que a gestão do conhecimento se guiará para os chamados "laboratórios de aprendizagem", os quais se utilizando das técnicas de gestão se adaptarão às mudanças ambientais com maior rapidez e flexibilidade. Assim, os autores definem quatro aspectos críticos:

- solução de problemas (nas operações existentes);

- integração de conhecimento interno (entre funções e projetos);

- inovação e experimentação (para construção do futuro); e

- integração dos fluxos externos de informação.

A Figura 2 demonstra a interação destes aspectos.

Para gerir de forma eficiente o conhecimento, são necessários métodos que influenciem os ativos intelectuais da organização a fim de promover seu desenvolvimento, para tanto, seis processos essenciais foram elaborados por Probst, Raub e Romhardt (2002) para a gestão do conhecimento nas organizações. $\mathrm{O}$ Quadro 2 abaixo explicita tais processos.

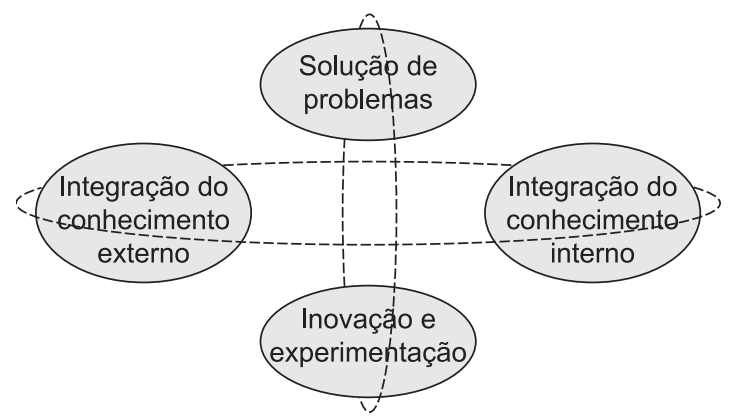

Figura 2. Elementos críticos do conhecimento. Fonte: Leonard e Barton (1992).

\section{A aprendizagem organizacional}

A aprendizagem e o conhecimento são conceitos interligados dentro de um processo de reforço mútuo, pois, a aprendizagem, no ponto de vista do processo, produzirá novos conhecimentos, novoso conteúdos, o que acarretará em um impacto no processo da aprendizagem futura, Steil e Pacheco (2008).

Assim, a Aprendizagem Organizacional é o processo que objetiva a constante criação e disseminação do conhecimento organizacional por meio de métodos que facilitem a transformação do conhecimento tácito em explícito, além de tornar a empresa extremamente flexível e adaptável a mudanças do ambiente externo, Silveira (2004).

Dentre as inúmeras definições descritas são listadas no Quadro 3, as mais relevantes sobre o tema.

Já Fleury e Oliveira Jr. (2001) definem que é por intermédio do processo de aprendizagem, o qual está intimamente ligado à gestão do conhecimento nas empresas, que se pode desenvolver as competências necessárias ao posicionamento estratégico. Com isso o processo de aprendizagem organizacional pode ocorrer em três níveis:

- Nível individual: lida com as emoções positivas ou negativas do indivíduo.

- Nível grupal: processo social compartilhado pelos indivíduos que compõem os diferentes grupos dentro da organização.

- Nível organizacional: a partir dos processos de aprendizagem individual e de partilha entre grupos tornam-se institucionalizados, expressando-se em estruturas, regras, procedimentos e elementos simbólicos. As organizações desenvolvem uma

Quadro 2. Os processos essenciais da gestão do conhecimento.

\begin{tabular}{|c|c|}
\hline Identificação do conhecimento & $\begin{array}{l}\text { Analisar e descrever o ambiente de conhecimento da empresa, } \\
\text { assegurando a transparência interna e externa das informações. }\end{array}$ \\
\hline Aquisição do conhecimento & $\begin{array}{l}\text { Captar conhecimento do meio externo, por meio de clientes, fornecedores, } \\
\text { concorrentes, etc. ou também comprar conhecimento por meio do } \\
\text { recrutamento de especialistas. }\end{array}$ \\
\hline Desenvolvimento do conhecimento & $\begin{array}{l}\text { Gerar novas habilidades, ideias, processos e produtos captados pelo } \\
\text { processo criativo. }\end{array}$ \\
\hline Distribuição do conhecimento & $\begin{array}{l}\text { É o processo de compartilhar o conhecimento que já se encontra presente } \\
\text { na organização, devem ser observadas duas questões-chave: } \\
\text { Quem deve saber quanto sobre o que, ou ser capaz de fazer o que e em que } \\
\text { nível? } \\
\text { Como podemos facilitar o compartilhamento e a distribuição do } \\
\text { conhecimento? }\end{array}$ \\
\hline Utilização do conhecimento & $\begin{array}{l}\text { Aplicar e desenvolver habilidades e ativos de conhecimento plenamente em } \\
\text { benefício da organização. }\end{array}$ \\
\hline Retenção do conhecimento & $\begin{array}{l}\text { É o processo de selecionar, armazenar e atualizar o conhecimento } \\
\text { regularmente, por meio de experiências, documentos e informações. }\end{array}$ \\
\hline
\end{tabular}

Fonte: Prosbst, Raub e Romhardt (2002). 
Quadro 3. Descrições mais relevantes acerca da aprendizagem organizacional.

Dentre as inúmeras definições descritas são listadas abaixo as mais relevantes sobre o tema:

\begin{tabular}{|c|c|}
\hline Autor & Definição \\
\hline Argyris (1977) & É o processo de detecção e correção de erros. \\
\hline Fiol; Lyles (1985) & $\begin{array}{l}\text { É o processo de aperfeiçoamento das ações por meio de aumento do conhecimento e da } \\
\text { compreensão. }\end{array}$ \\
\hline Levitt; March (1988) & $\begin{array}{l}\text { É o resultado de um processo de codificação de inferências baseadas na história em } \\
\text { rotinas de orientação do comportamento da organização. }\end{array}$ \\
\hline Huber (1991) & $\begin{array}{l}\text { Uma organização aprende quando, por meio do seu processamento de informações, } \\
\text { ela aumenta a probabilidade de que suas ações futuras promovam um acréscimo de } \\
\text { desempenho. }\end{array}$ \\
\hline Stata (1989) & $\begin{array}{l}\text { O aprendizado ocorre por meio do compartilhamento de idéias, conhecimentos e modelos } \\
\text { mentais, fundamentando-se no conhecimento e experiências do passado. }\end{array}$ \\
\hline Kim (1993) & $\begin{array}{l}\text { Aprendizagem Organizacional é definida como o incremento da capacidade } \\
\text { organizacional de realizar uma ação efetiva. }\end{array}$ \\
\hline Probst; Buchel (1997) & $\begin{array}{l}\text { Aprendizagem Organizacional é o processo pelo qual o conhecimento e os valores básicos } \\
\text { de uma organização mudam, buscando o aprimoramento da habilidade de solução de } \\
\text { problemas e da capacidade de ação. }\end{array}$ \\
\hline Fleury; Fleury (1997) & $\begin{array}{l}\text { A aprendizagem é um processo de mudança, resultante de prática ou experiência anterior, } \\
\text { onde pode ocorrer ou não uma modificação perceptível no comportamento. }\end{array}$ \\
\hline
\end{tabular}

memória com capacidade de reter e recuperar informações.

Senge (1998) define a organização que aprende como um ambiente em que as
"pessoas expandem continuamente sua capacidade de criar os resultados que realmente desejam, onde se estimulam padrões de pensamento novos e abrangentes, a aspiração coletiva ganha liberdade e onde as pessoas aprendem continuamente a aprender juntas".

Para que ocorra tal desenvolvimento, Senge desenvolveu as cinco disciplinas básicas pelas quais o ciclo de aprendizado é ativado, são elas:

- Domínio pessoal: O foco desta disciplina é o individuo, fomentando neste o desejo e a capacidade de aprender, por meio da busca pelo autoconhecimento, criando um ambiente empresarial que estimulará todos os participantes a alcançar as metas escolhidas.

- Visão compartilhada: É função dos líderes tornar objetivos da organização concretos e legítimos, desenhando imagens do futuro, de modo que estimule o engajamento do grupo que se dedicará não por obrigação, mas por comprometimento e vontade própria.

- Modelos mentais: É um sistema de valores baseado nas descobertas pessoais, que ajudam a melhorar a imagem que cada um tem do mundo, com o intuito de verificar ideias, atos e decisões, que influenciam o modo de como a organização será gerida.
- Aprendizado em equipe: Visando alcançar a ação coordenada, o aprendizado em equipes une as aptidões coletivas desenvolvendo inteligência, dialogo proposição de ideias, obtendo assim capacidades de trabalho maiores que a simples soma dos talentos individuais.

- Pensamento sistêmico: Ele se integra às demais disciplinas por meio de um modelo conceitual, neste há instrumentos que visam melhorar continuamente os processos de aprendizagem, tornando as ações de aprendizado inter-relacionadas às demais disciplinas.

\section{Ferramentas de pesquisa}

- O teste sociométrico e o sociograma

Moreno em 1974 desenvolveu a socionomia que propõe o estudo do tratamento dos indivíduos e suas relações interpessoais em grupos. O teste sociométrico é uma técnica que consiste, na aplicação de um questionário pessoal das escolhas preferenciais dos indivíduos entre si, relacionados às situações que se deseja pesquisar, permitindo assim, determinar o grau no qual os indivíduos são aceitos ou rejeitados em um grupo, descobrir as relações entre os indivíduos e revelar sua estrutura.

O sociograma é a representação gráfica das relações identificadas pelo teste sociométrico, caracterizando assim um mapa dos canais por onde o conhecimento é transferido, permitindo a visualização da coesão do grupo, Santos (2004). Com o sociograma podem-se obter evidências da disposição de cada membro em seu grupo, identificando os que têm maior (estrela) e 
menor (periférico) potencial de liderança, os rejeitados, os isolados, (ROCHA, 2003).

- Organizational Learning Survey

Para avaliar a capacidade de aprendizagem de uma organização, Goh e Richard (1997) desenvolveram um instrumento de pesquisa denominado Organizational Learning Survey $(O L S)$, que identifica as cinco dimensões que agrupam as melhores características e práticas de gestão consideradas essenciais para o desenvolvimento da aprendizagem organizacional:

- Claridade da finalidade e da missão: A organização como um todo necessita ter uma finalidade claramente definida, para que se compreenda a forma como cada indivíduo contribui com seu trabalho para a realização da missão da organização.

- Compromisso e Empowerment da liderança: Os líderes são considerados um elemento essencial para promover o clima de aprendizagem, eles devem criar um clima de confiança e de aproximação entre os indivíduos, incentivar a busca pelo conhecimento e demonstrar que as falhas são uma parte do processo de aprendizagem.

- Experimentação e recompensas: A estrutura e os sistemas devem suportar e promover oportunidades para a experimentação de novas ideias de trabalho e processos inovadores.

- Transferência do conhecimento: As informações adquiridas e distribuídas devem relacionar-se aos problemas e às oportunidades da organização, para transferir o conhecimento por meio dos limites departamentais para o ambiente externo.

- Resolver problemas do Teamwork: As estruturas e os sistemas na organização devem incentivar o trabalho em grupo para solucionar problemas com autonomia, reduzindo a dependência destes na gerência superior.

\section{A pesquisa}

O objeto de estudo selecionado foi o Laboratório Nacional de Astrofísica - LNA, pertencente ao Ministério da Ciência e Tecnologia - MCT. Ele foi o primeiro Laboratório Nacional implantado no Brasil em 1985, desenvolvendo pesquisas nas áreas de astronomia, astrofísica, bem como o desenvolvimento de componentes para satélites e telescópios.

A pesquisa foi construída em torno da aplicação do teste em 59 dos 66 funcionários, sendo que seus nomes foram substituídos por números para preservar sua individualidade.

O questionário sociométrico buscou identificar as relações sociais e de trabalho entre os indivíduos de diversas áreas dentro da instituição, verificando os principais indivíduos detentores do conhecimento. Foram inseridas questões sobre os locais de socialização, para possível melhora em sua disposição, além de questões sobre as formas de comunicação a fim de se verificar como o conhecimento é transmitido;

A aplicação do questionário OLS buscou calcular o potencial de aprendizagem organizacional, sendo constituído de 21 itens, validado com confiabilidade de 0,90 (alfa de Cronbach), os quais melhor capturam todas as cinco dimensões descritas anteriormente.

\section{Análise dos resultados}

Foram realizadas nove etapas para analisar os resultados obtidos nesta pesquisa, a primeira etapa é uma contextualização sobre o perfil dos respondentes, a segunda e terceira etapas são análises quanto aos resultados do teste sociométrico, a quarta e quinta etapas tratam da preparação dos dados e definição dos potenciais (PAPO) quanto ao questionário OLS e as demais etapas que constituem as análises estatísticas e conclusões preliminares.
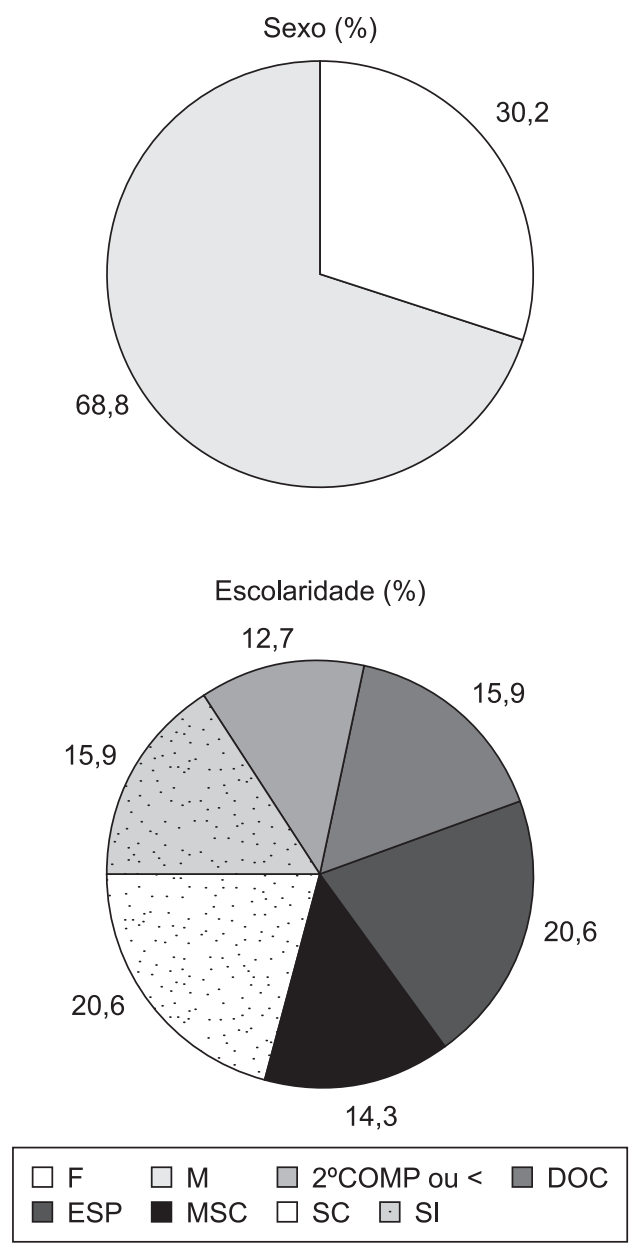

Figura 3. Gráfico de pizza para sexo e escolaridade. Fonte: Pesquisa. 
$1{ }^{\text {a }}$ Etapa - Perfil dos respondentes: Analisando-se os dados demográficos, identificou-se que a média de idade dos respondentes é de 40 anos; os anos de trabalho na organização são em média de 11 anos, bem como os anos nos setores específicos com média de 10 anos. A Figura 3 informa as distribuições de sexo e escolaridade da instituição.

Observa-se uma predominância masculina dentro do LNA com cerca de $70 \%$, as escolaridades com maior porcentagem se encontram nos indivíduos com superior completo e especialização ambos com aproximadamente $20 \%$. Nas demais escolaridades nota-se que aproximadamente $13 \%$ possuem segundo grau completo ou menor, o que denota um bom nível de instrução dentro do laboratório.

$\mathbf{2}^{\mathbf{0}}$ etapa - Análise Sociométrica: Ao analisar os dados colhidos, percebe-se:

Para o teste sociométrico de afinidade, quando solicitado aos entrevistados para indicar até três colegas de trabalho para questões pessoais, foram observados os seguintes resultados:
- o funcionário número 54 é tido como uma liderança informal, denominada "estrela", dentro do laboratório, acumulando um total de dez indicações;

- a liderança secundária foi observada com o funcionário número 19, acumulando um total de seis indicações;

- os números 38 e 53 com cinco indicações, os números 8,27 e 58 com quatro indicações, são considerados indivíduos-chave pelo seu nível de socialização, mas não são caracterizados como lideranças;

- os demais funcionários receberam três indicações ou menos, considerados "seguidores";

- os funcionários não indicados são os números $1,3,7,21,22,25,28,33,36,37,40,44,48$, 59 , sendo considerados isolados do grupo social dentro do laboratório, denominados assim como "periféricos".

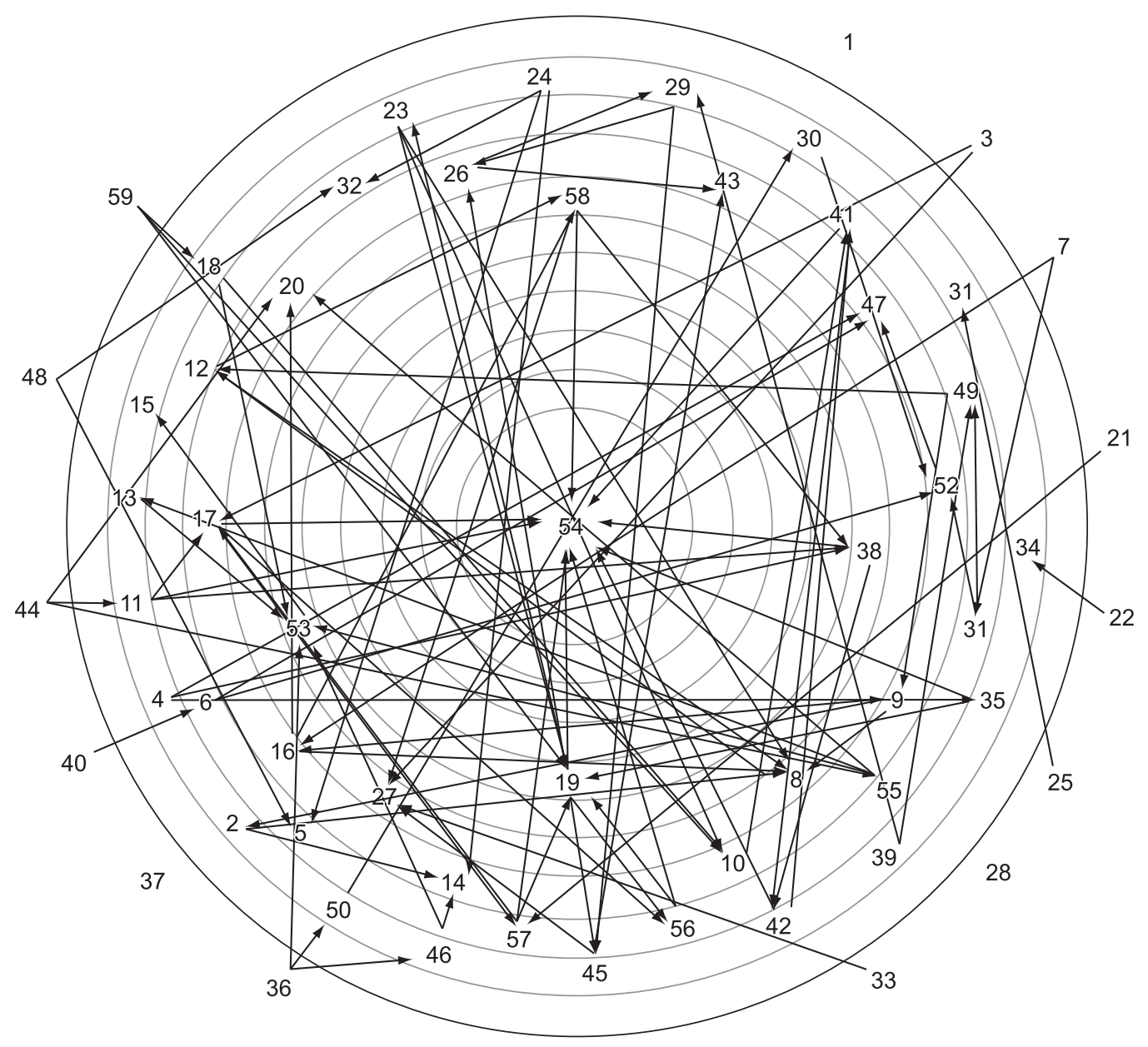

Figura 4. Sociograma de afinidade. Fonte: Pesquisa realizada. 
As relações pessoais e de trabalho estão demonstradas nos sociogramas (Figura 4):

Para o teste sociométrico de relações de trabalho, já se evidencia um novo quadro quando os entrevistados foram solicitados a indicar até três colegas para questões relativas a suas atividades dentro do laboratório. Foram observados os seguintes resultados:

- o funcionário número 58 é tido como uma liderança formal, denominada "estrela", dentro do laboratório, acumulando um total de dezesseis indicações;

- a liderança secundária foi observada nos funcionários de número 8, com onze indicações, e de número 12, com dez indicações, considerados indivíduos-chave pelo seu nível de socialização, mas não são caracterizados como lideranças;

- os demais funcionários relacionados possuíam oito indicações ou menos, sendo que tal quantidade não é caracterizada como liderança, são considerados "seguidores";
- os indivíduos não relacionados foram os números, 3, 7, 10, 13, 17, 18, 21, 23, 25, 26, 28, 29, 33, 34, 36, 39, 44, 48, 50, 56, 59, sendo considerados isolados ou "periféricos" pelo grupo de trabalho.

As relações pessoais e de trabalho estão demonstradas nos sociogramas (Figura 5):

$3^{\text {o }}$ etapa - Análise dos locais e meios de comunicação: A pesquisa identificou que o LNA possui uma estrutura concentrada e muito próxima entre suas dependências, o que permite aos indivíduos se encontrarem na maioria das vezes pessoalmente. O local mais utilizado para este encontro é a cafeteria localizada no centro entre os escritórios e os laboratórios.

A pesquisa também abordou as formas de comunicação existente entre os indivíduos, a forma verbal (pessoalmente ou por telefone) foi a mais constatada (87\%), seguida pelo correio eletrônico (9\%), formas físicas de comunicação foram pouco citadas (4\%).

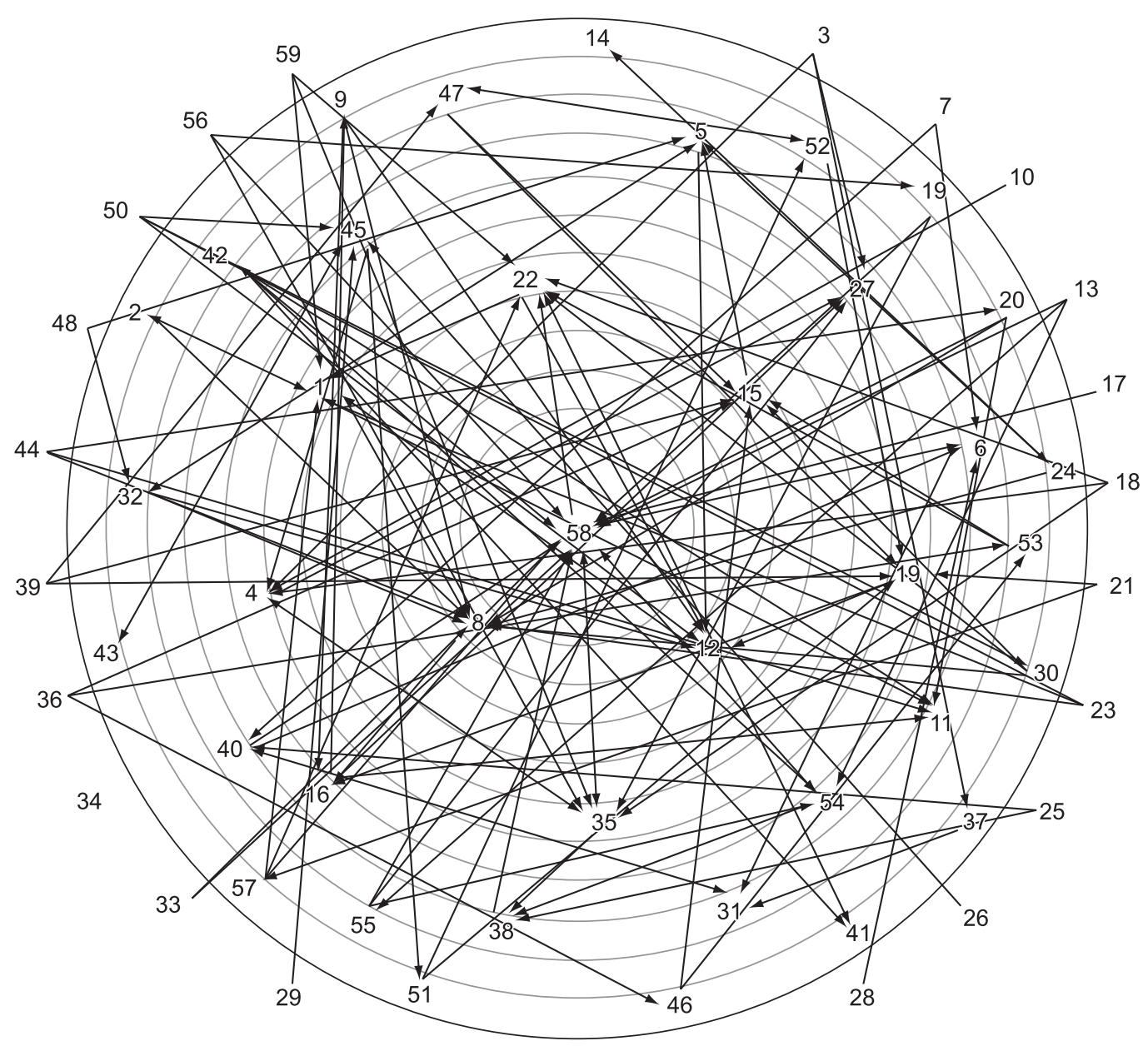

Figura 5. Sociograma de relações de trabalho. Fonte: Pesquisa realizada. 


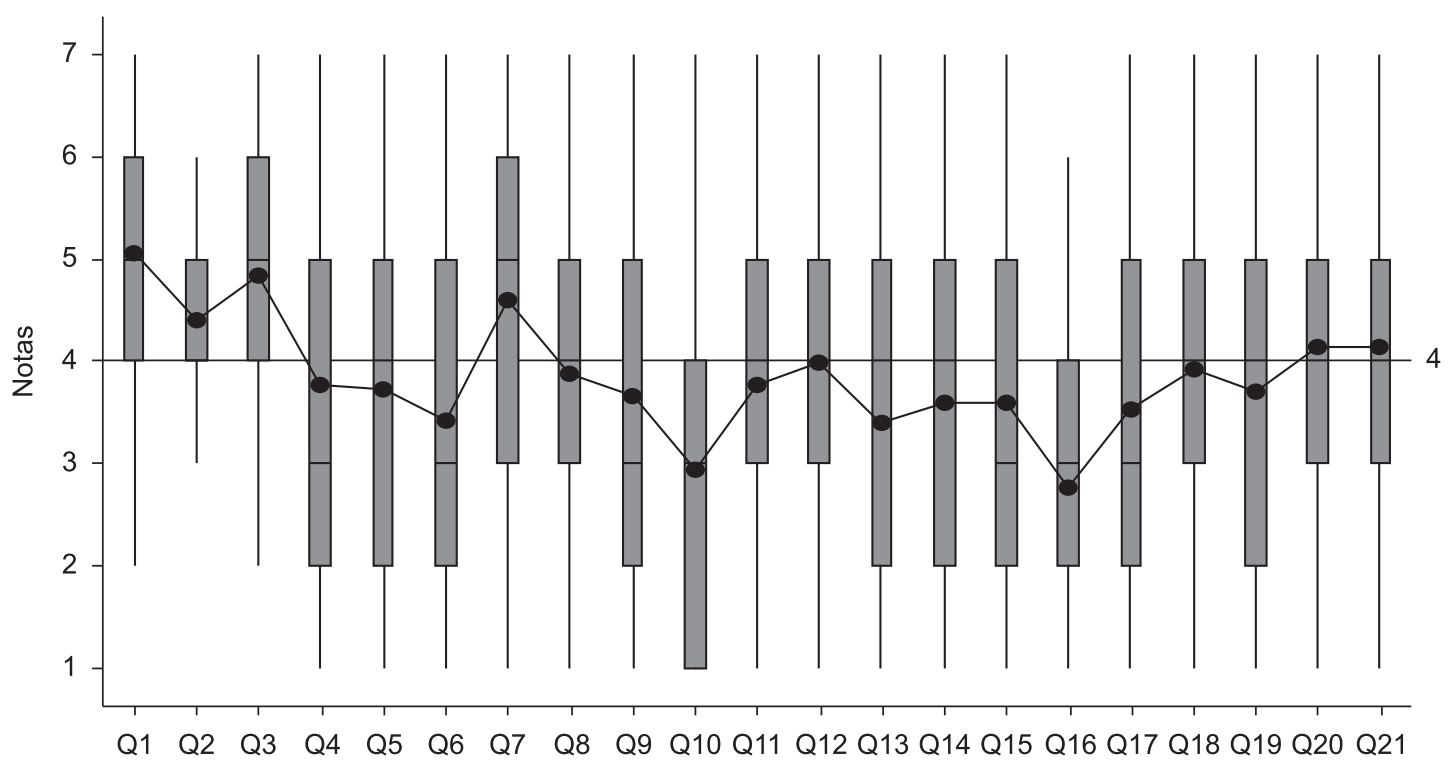

Figura 6. Gráfico Boxplot para os resultados. Fonte: Pesquisa.

$4^{\text {a }}$ Etapa - Preparação dos dados da OLS: Em seguida, os dados foram trabalhados para a eliminação dos outliers, por meio do gráfico boxplot. Neste gráfico identificamos as 21 questões no eixo $\mathrm{x}$, as notas no eixo y, a distribuição dos dados por questão, suas médias e medianas (Figura 6).

5 Etapa - Definição dos potenciais: Foi analisado na plataforma Minitab o Potencial de Aprendizagem organizacional (PAPO) do LNA e de seus respectivos setores, segundo o modelo de Angelim (2003), no qual o cenário em relação à $\mathrm{AO}$ é mais favorável quanto mais próximos de 7 estiverem os valores e, quanto mais próximos de 1 , mais desfavorável o cenário é à $\mathrm{AO}$. O potencial é o calculo da média das respostas (Tabela 1).

Também foi calculado o potencial geral do LNA em função das cinco dimensões apresentadas por Goh \& Richard, conforme Tabela 2.

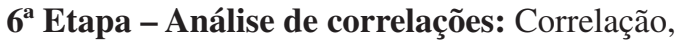
segundo Rencher (2002), é o fator estatístico que mede o quanto os dados estão relacionados entre si quanto à força (quanto mais próximo de 1) e sentido (positivo e negativo), pois, se as variáveis estão no mesmo sentido, cada resposta dada afetará a seguinte e a força irá informar de quanto será este impacto.

Correlações entre as 21 questões: ao se analisar as correlações entre as questões, percebeu-se que em quase todos os casos a correlação existe e é significante e forte em sentido positivo, ou seja, todas as respostas influenciam umas às outras, tal resultado sugere uma análise multivariada das respostas, o que será apresentado nas próximas etapas.

Correlações entre PAPO, anos na organização, anos na área e idade: $o$ índice $P$-value abaixo de
0,05 indica se há correlação. Neste caso, os resultados obtidos pela plataforma Minitab ${ }^{\circledR}$ foram:

- $\quad$ PAPO e Anos Organização P-value 0,141;

- PAPO e Anos Área $P$-value 0,195; e

- PAPO e Idade $P$-value 0,019 .

Tabela 1. Definição do PAPO.

\begin{tabular}{lc}
\hline Instituição & PAPO \\
\hline LNA & 3,852 \\
Setores & \\
Diretoria & 3,864 \\
CAC & 4,164 \\
COTE & 4,490 \\
CAD & 3,051 \\
COPD & 3,476 \\
SEMI & 3,333 \\
SSL & 4,533 \\
\hline
\end{tabular}

Fonte: Pesquisa.

Tabela 2. Potenciais das dimensões.

\begin{tabular}{lc}
\hline \multicolumn{1}{c}{ Dimensões } & Potenciais \\
\hline Claridade da finalidade e da missão & 3,805 \\
Compromisso e Empowerment & 3,893 \\
da liderança & \\
Experimentação e recompensas & 3,987 \\
Transferência do conhecimento & 3,733 \\
Resolver de problema do & 3,830 \\
Teamwork e do grupo & \\
\hline
\end{tabular}

Fonte: Pesquisa. 
Percebeu-se que o tempo dos indivíduos na organização ou em seus setores não possui correlação com o PAPO dos indivíduos, porém, quando analisada a idade há uma correlação significante, negativa, contudo, fraca.

No LNA, as faixas de idade variam entre 18 e 64 anos, assim, com base nos resultados podemos afirmar que a idade do indivíduo tem certa influência em sua pré-disposição para a aprendizagem organizacional, ou seja, quanto mais jovem o indivíduo mais pré-disposto ele estará para assumir um comportamento de aprendizagem.

$7^{a}$ Etapa - Análise ANOVA: Foi realizada a análise de variância, que mede a dispersão dos dados ao redor da média, utilizada para verificar se a média entre os potenciais são iguais quanto aos critérios demonstrados na Tabela 3, Rencher (2002).

Quanto à análise das médias do $\mathrm{PAPO}$, percebe-se que a do setor CAD possui uma média diferente e inferior aos setores CAC, COTE e SSL, que possuem melhores potenciais. Porém, todos os setores possuem potenciais relativamente baixos, sendo igual ou inferior a média geral.

Quanto ao critério do tipo de vínculo com a instituição, percebe-se que o melhor potencial se encontra no tipo de vínculo 6 , correspondente aos bolsistas, e verificou-se que suas médias são diferentes em relação aos demais tipos de vínculos encontrados, servidores da própria instituição ou cedidos por outras

Tabela 3. Análise dos critérios em relação ao PAPO.

\begin{tabular}{ll}
\hline \multicolumn{1}{c}{ ANOVA } & Resultados \\
\hline PAPO vs. Setor & P-value 0,012 \\
PAPO vs. Vínculo & P-value 0,000 \\
PAPO vs. Escolaridade & P-value 0,002 \\
PAPO vs. Sexo & P-value 0,698 \\
\hline
\end{tabular}

Fonte: Pesquisa.

Tabela 4. Análise por componente principal.

\begin{tabular}{ll}
\hline \multicolumn{1}{c}{ PCA } & Resultados \\
\hline PC1 vs. Setor & P-value 0,013 \\
PC1 vs. Vinculo & P-value 0,000 \\
PC1 vs. Escolaridade & P-value 0,002 \\
PC1 vs. Sexo & P-value 0,591 \\
\hline
\end{tabular}

Fonte: Pesquisa. instituições. Contudo, novamente observaram-se baixos valores nos potenciais no geral.

Quanto ao critério escolaridade, notou-se que as médias dos potenciais são diferentes na maioria dos casos, sendo que o menor potencial está nos indivíduos com especialização e o maior potencial está nos indivíduos com superior completo ou incompleto. $\mathrm{Na}$ análise quanto ao sexo, não há diferença entre as médias, apresentando valores próximos.

$8^{\text {a }}$ Etapa - Análise de componentes principais: A análise de componentes principais consiste essencialmente em reescrever as coordenadas das amostras em outro sistema de eixo mais conveniente para a análise dos dados.

As n-variáveis da pesquisa geram, por suas combinações lineares, n-componentes principais, cujas características são a obtenção em ordem decrescente de máxima variância, ou seja, a componente principal 1 detém mais informação estatística que a componente principal 2 e assim por diante. (NETO; MOITA, 1997).

Este método permite a redução dimensional das amostras, pois, embora a informação estatística seja a mesma dos $\mathrm{n}$ componentes principais, é comum obter-se em apenas duas ou três dos primeiros componentes mais que $90 \%$ desta informação. Assim, todos os testes foram refeitos e observaram-se consistências nos resultados obtidos nas análises de variância, como mostra a Tabela 4.

Dessa forma, todos os testes foram repetidos para averiguar os resultados em relação ao componente principal. Após os testes, foram confirmadas todas as análises, observando-se os mesmos resultados obtidos com a análise de variância.

$9^{\text {a }}$ Etapa - Análise MANOVA: Devido ao conjunto de dados e variáveis ser constituído de um grande número, a existência de uma forte correlação e interdependência identificada nos 21 itens do questionário, tornou-se necessária à análise multivariada. Para obter informações úteis dessa massa de dados, para investigação da dependência e relações estruturais entre variáveis, além de verificar as diferentes médias de PAPO em relação aos critérios setor, vínculo, escolaridade e sexo (PAIVA, 2006).

MANOVA para Setor: Neste caso identificou-se a existência de pouca diferença entre as médias, pois somente o teste de Lawley-Hotelling apontou uma diferença sensível nas questões 5, 6, 11, 14, 16,18 e

Tabela 5. MANOVA para Setor.

\begin{tabular}{lccccc}
\hline \multicolumn{1}{c}{ Critérios } & Estatística & Approx F & Nym & Denom & P \\
\hline Wilks' & 0,04392 & 1,226 & 126 & 216 & 0,095 \\
Lawley-Hoteling & 4,81076 & 1,311 & 126 & 206 & $\mathbf{0 , 0 4 3}$ \\
Pilai's & 2,24078 & 1,164 & 126 & 246 & 0,158 \\
Roy's & 2,38159 & & & & \\
\hline
\end{tabular}

Fonte: Pesquisa. 
Tabela 6. MANOVA para Vínculo.

\begin{tabular}{lccccc}
\hline \multicolumn{1}{c}{ Critérios } & Estatística & Approx F & Nym & Denom & P \\
\hline Wilk' & 0,25024 & 1,903 & 42 & 80 & $\mathbf{0 , 0 0 7}$ \\
Lawley-Hoteling & 2,13053 & 1,978 & 42 & 78 & $\mathbf{0 , 0 0 5}$ \\
Pillai's & 0,96639 & 1,825 & 42 & 82 & $\mathbf{0 , 0 1}$ \\
Roy's & 1,58403 & & & & \\
\hline
\end{tabular}

Fonte: Pesquisa.

Tabela 7. MANOVA para Escolaridade.

\begin{tabular}{lccccc}
\hline \multicolumn{1}{c}{ Critérios } & Estatística & Approx $\mathbf{F}$ & Nym & Denom & P \\
\hline Wilks' & 0,08086 & 1,189 & 105 & 185 & 0,153 \\
Lawley-Hoteling & 3,39264 & 1,144 & 105 & 177 & 0,215 \\
Pillai's & 1,93422 & 1,232 & 105 & 205 & 0,104 \\
Roy's & 1,21945 & & & & \\
\hline
\end{tabular}

Fonte: Pesquisa.

Tabela 8. MANOVA para Sexo.

\begin{tabular}{lccccc}
\hline \multicolumn{1}{c}{ Critérios } & Estatística & Approx F & Nym & Denom & P \\
\hline Wilks' & 0,59852 & 1,310 & 21 & 41 & 0,225 \\
Lawley-Hoteling & 0,67079 & 1,310 & 21 & 41 & 0,225 \\
Pillai's & 0,40148 & 1,310 & 21 & 41 & 0,225 \\
Roy's & 0,67079 & & & \\
\hline
\end{tabular}

Fonte: Pesquisa.

20, correspondentes no geral às dimensões de trabalho em grupo e à clareza de missão da organização.

Os resultados dos testes podem ser observados na Tabela 5, para tanto, o cálculo estatístico de F segue os parametros de $\mathrm{S}=6 ; \mathrm{M}=9,0 ; \mathrm{N}=17,0$.

MANOVA para Vínculo: Foi verificada uma grande diferença entre os potenciais em relação ao tipo de vínculo, previamente identificada na análise de variância dos respondentes, os vínculos 1 e 2, servidores da própria instituição ou cedidos por outras instituições, possuem menor potencial em relação ao vínculo 6 , bolsistas.

Os resultados dos testes podem ser observados na Tabela 6, para tanto, o cálculo estatístico de F segue os parametros de $\mathrm{S}=2 ; \mathrm{M}=9,0 ; \mathrm{N}=19,0$.

MANOVA para Escolaridade e Sexo: Quando testadas as médias em relação às respostas dos itens, percebe-se que não há diferenças significativas quanto ao nível de aprendizagem nos critérios escolaridade e sexo. Porém, no critério escolaridade, os testes de ANOVA e PCA identificaram diferenças significativas, portanto, este critério deverá ser reavaliado posteriormente.

Os resultados dos testes podem ser observados na Tabela 7, para tanto, o cálculo estatístico de F, para a variável Escolaridade, segue os parametros de $\mathrm{S}=6$; $\mathrm{M}=9,0 ; \mathrm{N}=17,0$.

Para tanto, o cálculo estatístico de $\mathrm{F}$, para a variável Sexo, segue os parâmetros de $S=6 ; M=9,0 ; N=17,0$ (Tabela 8).

\section{Considerações finais}

Com base nos resultados do teste sociométrico, percebe-se que o LNA possui um arranjo de grupos com grandes problemas quanto aos requisitos da Gestão do Conhecimento e da Aprendizagem Organizacional no que tange os aspectos dos quatro modos de conversão, principalmente quanto à socialização e à externalização do conhecimento, bem como, ao desenvolvimento de um ambiente favorável à comunicação e geração de modelos mentais.

O grande número de exclusões, 28 indivíduos no total, representa uma perda enorme de capital intelectual pela instituição, além disso, a forma extremamente tácita de comunicação faz com que ocorram falhas na transmissão de conhecimento, o que acarreta perdas incontáveis de ideias, experiências, inovações e novos conhecimentos.

Quanto à OLS, ao calcular os potenciais de aprendizagem, PAPO, pode-se identificar a situação de diversas áreas e setores da organização, vide análise de resultados, e focalizar nas áreas em que a melhoria é necessária para desenvolver o consenso para a tomada de decisão.

Os dados fornecido pela OLS ajudarão os membros da organização a encontrar o foco de ação e da missão, baseado nos modelos de Goh e Richars e na aprendizagem organizacional de Senge para desenvolver uma melhor transferência do conhecimento, do trabalho em grupo e da experimentação no sentido cognitivo 
e eventualmente comportamental, acarretando a melhoria do desempenho.

Portanto, com a aplicação destes testes e sua análise, pode-se afirmar que tanto a sociometria quanto a OLS provaram-se ferramentas úteis para diagnosticar as necessidades de implantação de um programa em gestão do conhecimento, pois identificam os pontos críticos tanto no ambiente de trabalho quanto no aspecto social da instituição, além de explicitar os aspectos gerenciais no ponto de vista da aprendizagem organizacional.

\section{Referências}

AMARAL, D. C.; ROZENFELD, H. Gerenciamento de conhecimentos explícitos sobre o processo de desenvolvimento de produto. In: CONGRESSO BRASILEIRO DE GESTÃO DE DESENVOLVIMENTO DE PRODUTO, 3., 2001, Florianópolis. Anais... Florianópolis: Universidade Federal de Santa Catarina, 2001.

ANGeliM, G. P. Potencial de aprendizagem organizacional e qualidade de gestão: um estudo multicaso em organizações públicas brasileiras. (Tese de Doutorado). Universidade de Brasília, Departamento de Administração. Brasília março. 2003.

ARGYRIS, C. Double loop learning in organization. Harvard Business Review, v. 55, n. 5, p. 115-125, 1977.

DRUCKER, P. Post: capitalist society. New York: Harper Business, 1993.

FIOL, C. M.; LYLES, M. A. Organizational learning. Academy of Management Review, v. 10, n. 4, p. 803-813, 1985.

FLEURY, A.; FLEURY, M. T. L. Aprendizagem e inovação organizacional: as experiências de Japão, Coréia e Brasil. 2 ed. São Paulo: Editora Atlas, 1997.

FLEURY, M. T. L.; OLIVEIRA JR., M. M. Gestão estratégica do conhecimento: integrando aprendizagem, conhecimento e competências. São Paulo: Atlas, 2001.

GOH, S.; RICHARDS, G. Benchmarking the learning capability of organizations. European Management Journal, v. 15, n. 5, p. 575-583, 1997.

HUBER, G. P. Organizational learning: the contributing process and the literatures. Organizational Science, v. 2, n. 1, p. 88-115, 1991.

JENSEN, M. B. et al. Forms of knowledge and modes of innovation. Research Policy, n. 36, p. 680-693, 2007.

KIM, D. G. The link between individual and organizational learning. Sloan Management Review, v. 35, n. 1, p. 37-50, 1993.

LABORATÓRIO NACIONAL DE ASTROFÍSICA - LNA. Plano Diretor 2006-2010: planejamento estratégico do LNA. Itajubá, 2006.

LEONARD-BARTON, D. The factory as learning laboratory. Sloan Management Review, v. 34, p. 23-38, 1992.
LEVITT, B.; MARCH, J. Organizational learning. Annual Review of Sociology, v. 14, p. 319-40, 1988.

MORENO, J. L. Psicoterapia de grupo e psicodrama: introdução à teoria e à práxis. São Paulo: Editora Mestre, 1974.

MOSCOVICI, F. Desenvolvimento interpessoal. Rio de Janeiro: Editora José Olympio, 1995.

MUNIZ, J. Modelo conceitual de gestão de produção baseado na gestão do conhecimento: um estudo no ambiente operário da indústria automotiva. Guaratinguetá, 2007. Tese (Doutorado em Engenharia de Produção) Universidade Estadual Paulista - UNESP.

NETO, J. M.; MOITA, G. C. Uma introdução à análise exploratória de dados multivariados. Piauí: Universidade Federal do Piauí, 1997.

NEVIS, E. G.; DIBELLA, A. J.; GOULD, J. M. Understanding organizations as learning systems. Sloan Management Review, v. 37, p. 73-85, 1995.

NONAKA, I.; TAKEUCHI, H. Criação de conhecimento na empresa. Rio de Janeiro: Editora Campus, 1997.

PAIVA, A. P. Metodologia de superfície de resposta e análise de componentes principais em otimização de processos de manufatura com múltiplas respostas correlacionadas. Itajubá, 2006. Tese (Doutorado) Universidade Federal de Itajubá - UNIFEI.

PROBST, G.; BUCHEL, B. S.T. Organizational learning. London: Prentice Hall, 1997.

RENCHER, A. C. Methods of multivariate analysis. 2 ed. New York: John Wiley \& Sons, 2002.

ROCHA, F. E. C. et al. Mapeamento das relações interpessoais em três assentamentos de reforma agrária de Unaí, MG. Cadernos de Ciência \& Tecnologia, v. 20, n. 2, p. 305-323, 2003.

SANTOS, R. C. A trajetória institucional e histórica da difusão do psicodrama pedagógico em Campinas: relatos orais sobre motivações vivenciais, contradições institucionais e perspectivas educacionais. Campinas, 2004. Dissertação (Mestrado em Educação) Universidade Estadual de Campinas - UNICAMP.

SENGE, P. M. A quinta disciplina: a arte e prática da organização que aprende. 2 ed. São Paulo: Editora Best Seller, 1998.

SILVEIRA, A. A. Gestão de conhecimento com ênfase na aprendizagem organizacional: um estudo multicaso no contexto bancários. Itajubá, 2004. Dissertação (Mestrado) - Universidade Federal de Itajubá - UNIFEI.

STATA, R. Organizational learning: the key to management innovation. Sloan Management Review, v. 30, n. 3, p. 63-74, 1989.

STEIL, A. V., PACHECO R. C. S. Aprendizagem organizacional e criação de conhecimento: em um instituto privado de ciência e tecnologia. Estratégia e Negócios, v. 1, n. 2, p. 25-42, 2008.

SVEIBY, K. E. The New Organization Wealth: managing and measuring knowledge-based assets. San Francisco: Berreth-Koehler Publishers, 1997.

TERRA, J. C.; KRUGLIANSKAS, I. Gestão do conhecimento em pequenas e médias empresas. Rio de Janeiro: Campus, 2003. 